\title{
Descripción osteológica de la taruca (Hippocamelus antisensis) (d’Orbigny, 1834): II. Esqueleto axial
}

\author{
Osteological description of the taruca (Hipocamelus antisensis) (d'Orbigny, 1834): \\ II. Axial skeleton
}

Celso Zapata Coacalla', Joel Iván Pacheco Curie ${ }^{2,3}$, José Carlos Arias Flores²

\section{Resumen}

\begin{abstract}
Hippocamelus antisensis (d'Orbigny 1834), llamado taruca comúnmente, es un ciervo que vive en Perú, Bolivia y Chile entre los 3500 a 5000 m, y es considerado como especie vulnerable. Se describe el esqueleto axial de la taruca con base a cuatro especímenes (dos machos y dos hembras), según autorización RDG N $158-2015$-SERFORDGGSPFFS. Las piezas óseas se obtuvieron conforme a la técnica anatómica, la descripción está de acuerdo con la Nómina Anatómica Veterinaria y las medidas a lo descrito por Von den Driesch. En el cráneo el occipital no tiene una protuberancia del occipital muy sobresaliente, el hueso frontal se caracteriza por su apófisis del asta y por presentar hasta tres forámenes supraorbitarios, el lagrimal presenta una fosa del lagrimal profunda y una fisura cubierta por cartílago, el hueso incisivo y maxilar son alargados, en el maxilar existe la presencia de un alveolo dentario para el canino, la sínfisis de la mandíbula permanece hasta la edad adulta, el vómer se proyecta hacia caudal relacionándose con el hueso palatino, presenta 7 vértebras cervicales, 13 torácicas, 7 lumbares, 5 sacras y 7 coccígeas y el esternón con 7 esternebras, presenta una apófisis xifoides pronunciada. Se concluye que la cabeza es alargada por las características de los huesos maxilar e
\end{abstract}

${ }^{1}$ Facultad de Medicina Veterinaria y Zootecnia, Universidad Nacional del Altiplano, Puno, Perú

${ }^{2}$ Estación de Centro de Investigación IVITA Maranganí, Facultad de Medicina Veterinaria, Universidad Nacional Mayor de San Marcos, Lima, Perú

${ }^{3}$ E-mail:jpachecoc@unmsm.edu.pe

Recibido: 25 de febrero de 2021

Aceptado para publicación: 12 de octubre de 2021

Publicado: 22 de diciembre de 2021

CLos autores. Este artículo es publicado por la Rev Inv Vet Perú de la Facultad de Medicina Veterinaria, Universidad Nacional Mayor de San Marcos. Este es un artículo de acceso abierto, distribuido bajo los términos de la licencia Creative Commons Atribución 4.0 Internacional (CC BY 4.0) [https:// creativecommons.org/licenses/by/4.0/deed.es] que permite el uso, distribución y reproducción en cualquier medio, siempre que la obra original sea debidamente citada de su fuente original 
incisivo, la presencia de forámenes y las características de las astas en el hueso frontal pueden ser utilizados para su identificación, la existencia de la fosa del lagrimal profunda y una fisura cubierta por cartílago, las coanas están divididas por el vómer.

Palabras clave: cráneo, vertebras, esternón, anatomía, osteología

\title{
Abstract
}

\begin{abstract}
Hippocamelus antisensis (d'Orbigny 1834), commonly called taruca, is a deer that lives in Peru, Bolivia and Chile between 3500 to $5000 \mathrm{~m}$, and is considered a vulnerable species. The axial skeleton of the taruca is described based on four specimens (two males and two females), according to authorization RDG $\mathrm{N}^{\circ}$ 158-2015-SERFOR-DGGSPFFS. The bone pieces were obtained according to the anatomical technique, the description is in accordance with the Veterinary Anatomical List and the measurements as described by Von den Driesch. In the skull, the occipital does not have a very prominent occipital protrusion, the frontal bone is characterized by its horn process and by presenting up to three supraorbital foramina, the lacrimal has a deep fossa and a fissure covered by cartilage, the incisor bone and maxilla are elongated, in the maxilla there is the presence of a dental socket for the canine, the symphysis of the mandible remains until adulthood, the vomer projects caudally relating to the palatine bone, it has 7 cervical vertebrae, 13 thoracic, 7 lumbar, 5 sacral and 7 coccygeal and the sternum with 7 sternebrae, presents a pronounced xiphoid process. It is concluded that the head is elongated due to the characteristics of the maxillary and incisor bones, the presence of foramina and the characteristics of the antlers in the frontal bone can be used for its identification, the existence of the deep lacrimal fossa and a covered fissure. by cartilage, the choanas are divided by the vomer.
\end{abstract}

Key words: skull, vertebrae, sternum, anatomy, osteology

\section{INTRODUCCIÓN}

En Sudamérica se encuentran cuatro géneros de cérvidos (Hippocamelus, Mazama, Odoecoileus y Pudu) (Agustina et al., 2020), Hippocamelus antisensis (d'Orbigny 1834), comúnmente llamado taruca o taruka, es un ungulado con marcas faciales características (Barrio, 2013), pudiéndose distinguir de otras especies de ciervos sudamericanos por sus dos astas, que se dividen solo una vez, cerca de la base, estando solo en los machos, así como por su mayor desarrollo muscular a diferencia de las hembras (Roe y Rees, 1976; Barrio, 2010). Estos cérvidos viven en áreas rocosas de los Andes peruanos, bolivianos y chilenos (Merkt, 1985; Barrio, 2010; Gazzolo y Barrio, 2016) entre 3500 y $5000 \mathrm{msnm}$. Estos animales son cazados, no solo por su carne sino además por los perjuicios causados en la agricultura y competir por alimento con rumiantes domésticos (Fuentes-Allende et al., 2016; Barrio et al., 2017), siendo por lo tanto, consideradas como "vulnerables" para la ecozona de la sierra peruana (SERFOR, 2016).

Las investigaciones anatómicas en los cérvidos son escasas (Weber y Gonzalez, 2003). Se tiene algunas descripciones del corzo (Capreolus capreolus L), especialmente del cráneo (Fandos y Orueta, 1991), de los dientes caninos (Chaplin y Atkinson, 1968) y dientes vestigiales (Ratcliffe, 1970), así como de anomalías mandibulares y dentales (Jackson, 1973). Por ello, este trabajo de investigación describe el esqueleto axial de la taruca (Hippocamelus antisensis) (d'Orbigny, 1834). 


\section{Materiales y Métodoos}

El estudio se realizó en la Estación Experimental del Centro de Investigación IVITA, sede Maranganí, de la Universidad Nacional Mayor de San Marcos, ubicada en Cusco, Perú. Se utilizaron cuatro ejemplares de taruca (dos hembras y dos machos) que fueron decomisados por caza ilegal en las provincias de Canchis y Canas, del departamento de Cusco y remitidos con Acta de Entrega N. ${ }^{\circ} 03-2018$ y forman parte de la colección de la Estación IVITA Maranganí, reconocida como Institución Nacional Depositaria de Material Biológico, mediante RDG N. ${ }^{\circ} 073$ 2017-SERFOR-DGGSPFFS. El presente estudio se ampara en la autorización de investigación RDG N. ${ }^{\circ}$ 158-2015-SERFORDGGSPFFS.

Para el proceso de obtención del esqueleto se procedió con la técnica descrita por Villarroel y Troncoso (2017). Los términos utilizados están de acuerdo con la Nómina Anatómica Veterinaria (Schaller, 1996). El procedimiento para medir las piezas óseas se hizo según lo descrito por Von den Driesch (1968). Para la medición de las piezas óseas se utilizó un calibrador vernier (Dial caliper ${ }^{\circledR}$, USA) de 150 x $0.02 \mathrm{~mm}$ y una regla milimetrada metálica (Matrix ${ }^{\circledR}$ ).

\section{Resultados}

\section{Cabeza}

Las principales medidas de la cabeza de la taruca se presentan en el Cuadro 1. La Figura 1 muestra el cráneo de estos especímenes.

Los huesos y su descripción son como sigue:

Occipital. Presenta sus tres porciones: basilar, lateral y escamosa. La protuberancia occipital ligeramente rugosa, dos apófisis yugulares cor-
Cuadro 1. Medidas de la cabeza de cuatro especímenes, dos machos y dos hembras, de taruca (Hippocamelus antisensis)

\begin{tabular}{lcc}
\hline Medidas (cm) & Macho & Hembra \\
\hline Largo total & 24.2 & 23.2 \\
Amplitud frontal & 10.9 & 8.1 \\
$\begin{array}{l}\text { Longitud basal } \\
\text { Longitud } \\
\text { condilobasal }\end{array}$ & 21.3 & 20.5 \\
$\begin{array}{l}\text { Amplitud } \\
\text { zigomática }\end{array}$ & 22.6 & 17.5 \\
\hline
\end{tabular}

tas, aplanadas e inclinadas hacia medial, y en la zona basilar sobresale en la superficie ventral dos tubérculos musculares prominentes.

Esfenoides. Hueso que forma la parte ventral del cráneo. En la parte dorsal una pequeña fosa y rostral sobresale una porción de hueso. Las alas son cortas. El hueso esfenoides se relaciona con dos forámenes, uno rostral equivalente al foramen redondo, orbital y troclear, y el caudal que corresponde al foramen oval.

Temporal. Presenta una apófisis cigomática para articularse con el hueso cigomático, es delgado y ligeramente recto a nivel de la superficie para articularse al cóndilo de la mandíbula. El tubérculo articular es poco prominente, la fosa mandibular poco profunda y la apófisis retroarticular delgada, la bulla timpánica es poco prominente.

Parietales. Forman parte de la pared lateral (la superficie externa ligeramente convexa) y el techo de la cavidad craneana (la superficie externa ligeramente aplanada). A nivel de la sutura con el hueso frontal se relacionan casi inmediatamente con la formación de las astas en los machos. Presentan dos crestas poco pronunciadas que se originan en los extremos de la protuberancia occipital y se dirigen hacia las astas. 


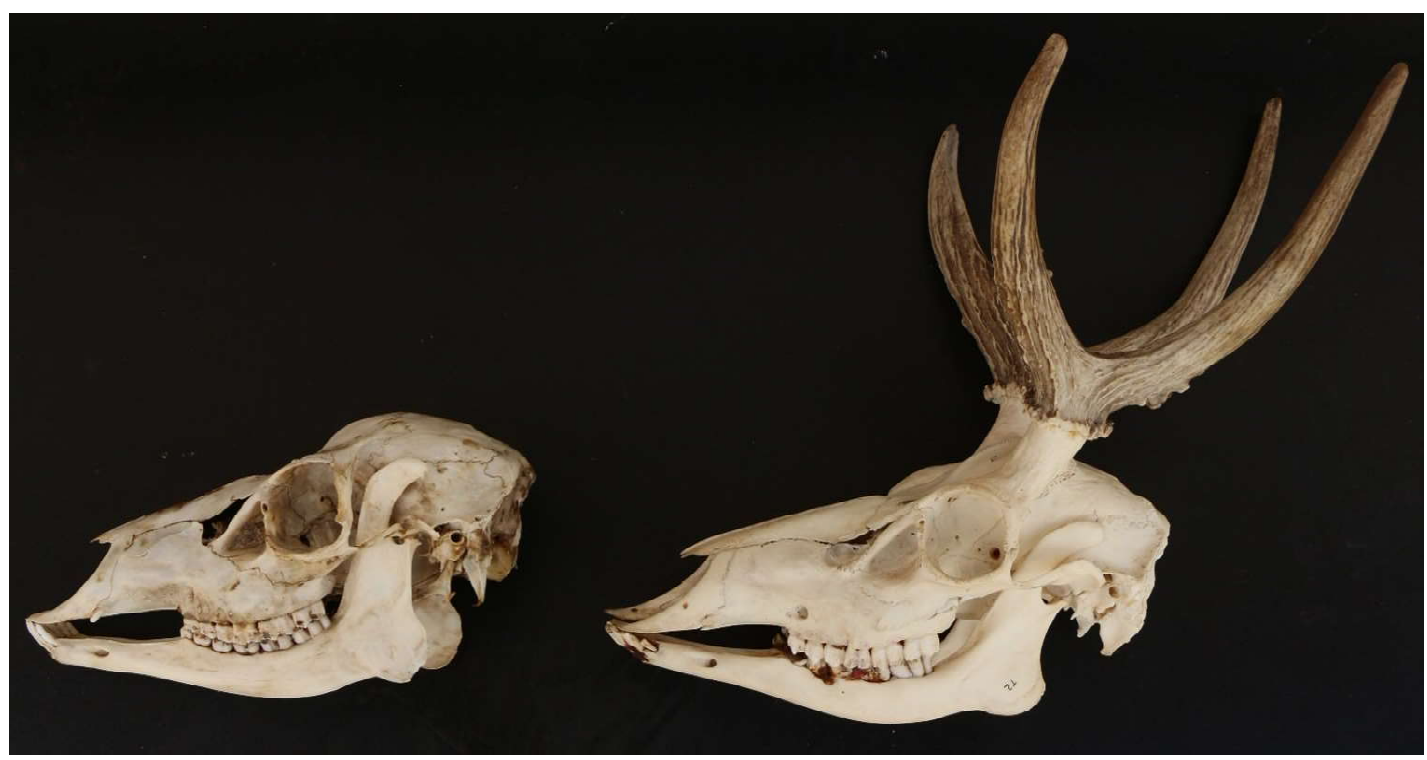

Figura 1. Fotografía de la cabeza de taruca hembra (Izq.) y macho (Der.)

Frontal. Es grande, y en los machos sobresale una apófisis compacta para el asta, muy cerca a la sutura con el parietal en su origen se dirige hacia caudal. De ahí se divide en dos ramas, siendo la anterior corta y curva, cuya punta se dirige hacia caudal, y la posterior más larga, ligeramente recta y también dirigido hacia caudal. En las hembras se logra observar un vestigio de las astas, poco sobresaliente. La apófisis cigomática completa, los agujeros supra orbitarios presentan uno bien definido y grande y los otros pequeños, suelen no ser iguales en ambos lados. A nivel de su borde ocular presenta una escotadura rugosa, la superficie anterior ligeramente cóncava dando cierto ángulo a la cara, en especial el límite entre la cavidad craneana y la cavidad nasal

Etmoides. Presenta una fosa etmoidal ligeramente oval y una cresta poco prominente.

Mandibula. Hueso completo con presencia de una forma estética con su cuerpo y rama mandibular, una sínfisis mandibular evidente.
El proceso coronoideo en forma de gancho y con dirección caudal, con proceso condilar estrecho, el ángulo mandibular prominente, delgado y dirigido hacia lateral, la fosa del masetero poco profunda. A nivel del borde alveolar presenta cuatro piezas dentarias de los cuales tres son incisivos y el cuarto es un canino modificado, diastema amplio. También presenta seis piezas dentarias que corresponde a molares y premolares y en su superficie oclusal las cúspides son bien pronunciadas.

Vomer. Hueso que se proyecta caudalmente sobresaliendo del hueso pteriogoideo. En su última porción en su borde dorsal se abre a manera de «Y» para articularse con el esfenoides.

Lagrimal. Hueso bastante desarrollado, en el borde orbital presenta dos forámenes, en la superficie facial presenta una profunda fosa del lagrimal, una fisura de forma trapezoidal, relacionando a los huesos frontal, nasal y maxilar con una abertura bastante amplia. 
Cuadro 2. Medidas óseas de las vértebras de cuatro especímenes, dos machos y dos hembras, de taruca (Hippocamelus antisensis) (promedios)

\begin{tabular}{|c|c|c|c|c|c|c|c|c|c|c|c|}
\hline \multirow{2}{*}{$\begin{array}{l}\text { Medida } \\
(\mathrm{cm})\end{array}$} & \multirow{2}{*}{ Atlas } & \multirow{2}{*}{ Axis } & \multicolumn{2}{|c|}{ Cervical } & \multicolumn{3}{|c|}{ Torácica } & \multicolumn{3}{|c|}{ Lumbar } & \multirow[t]{2}{*}{ Sacro } \\
\hline & & & $4^{\circ}$ & $7^{\circ}$ & $1^{\circ}$ & $4^{\circ}$ & $13^{\circ}$ & $1^{\circ}$ & $4^{\circ}$ & $6^{\circ}$ & \\
\hline GB & 7.3 & & & & & & & & & & 9 \\
\hline GL & 6 & & & & & & & & & & 12.4 \\
\hline $\mathrm{H}$ & 3.4 & 5.8 & & 7.4 & 9.2 & 9.6 & 5.7 & 5.7 & 5.9 & 10 & \\
\hline BPtr & & 5.1 & & 6.5 & & & & 8.7 & 12 & 5.5 & \\
\hline LCDe & & 7 & & & & & & & & & \\
\hline GLPa & & & 6 & & & & & & & & \\
\hline Bpacr & & & 3.8 & & & & & & & & \\
\hline Bpacd & & & 4.1 & & & & & & & & \\
\hline
\end{tabular}

GB: mayor amplitud sobre las alas

GL: mayor longitud

$\mathrm{H}$ : mayor altura

BPtr: la más grande amplitud del proceso transverso

LCDe: la mayor longitud en la región del cuerpo

GLPa: la mayor longitud desde el proceso articular craneal al proceso articular caudal

Bpacr: la mayor amplitud a través de los procesos articulares craneales

Bpacd: la mayor amplitud a través de los procesos articulares caudales

Maxilar. Presenta una forma alargada hacia rostral. Es evidente el agujero infraorbitario aproximadamente a nivel del primer premolar, un tubérculo facial poco prominente. El borde alveolar alberga a seis piezas dentarias, tres premolares y tres molares. A nivel caudal presenta una tuberosidad maxilar prominente y ganchoso. En la superficie palatina está presente el foramen palatino y forma parte del borde caudal y lateral de una gran fisura palatina, también el hueso maxilar origina los cornetes nasales ventrales. En los especímenes machos y hembras se encontró el alveolo dentario para el diente canino.

Nasal. Hueso largo convexo transversal y ligeramente escotado en la parte media, el extremo rostral termina en punta, forma una gran escotadura en conjunto con el hueso incisivo y algunos especímenes con el hueso maxilar, forma parte de los cornetes nasales dorsales.

Incisivo. El cuerpo es aplanado y sin alveolos, la apófisis nasal llega a relacionarse con el hueso nasal y en algunos especímenes no llega a relacionarse. La apófisis palatina es bastante larga.

Cigomático. Presenta apófisis para el frontal y el temporal. No forman un arco cigomático sobresaliente y curvo, participa de uno de los bordes de la fosa del lagrimal.

Palatino. A nivel de su borde medial comparte relación con el hueso vómer en toda su longitud y una apófisis pterigoidea no muy sobresaliente.

Pterigoideos. Su apófisis pterigoidea es muy sobresaliente y ganchosa. 


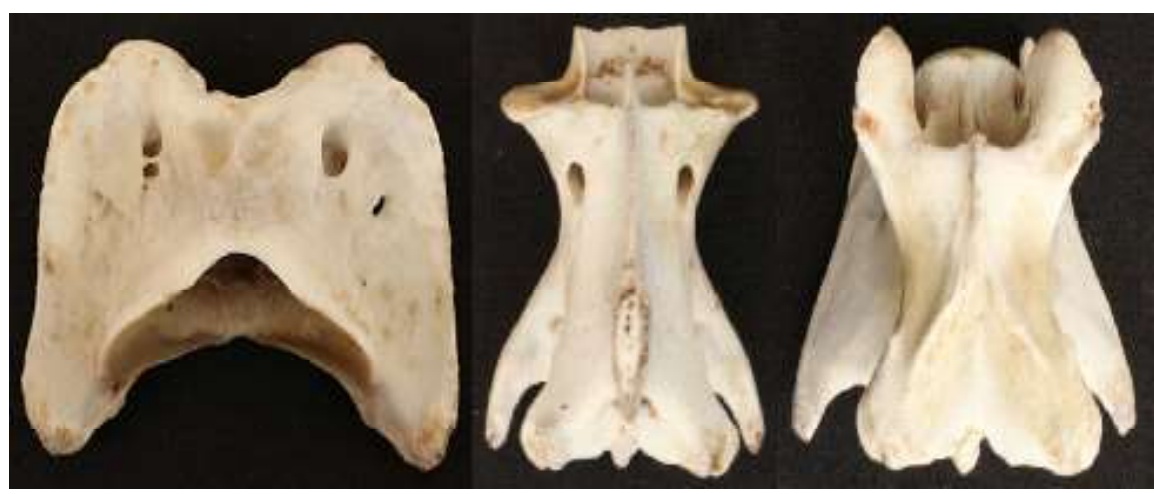

Figura 2. Detalle de vértebras cervicales de la taruca (Hippocamelus antisensis): Atlas (izquierda), Axis (centro) y C4 (derecha)

\section{Vértebras}

Las medidas promedio de las piezas óseas de las vértebras se presentan en el Cuadro 2. La descripción de las vértebras es la siguiente:

Atlas. Alas ligeramente horizontales poco prominentes, foramen alar incipiente, fosa alar poco profunda.

Axis. Espina poco prominente y no sobresale del cuerpo de la vértebra. Las apófisis articulares caudales presentan un sobresaliente a manera de apófisis mamilar, superficies articulares con el atlas están divididas ventralmente por una escotadura profunda, un diente poco sobresaliente y presenta un foramen transverso pequeño.

$1^{\circ}, 4^{\circ}$ y $7^{\circ}$ cervicales. Huesos completos con relieves bien definidos, ligeramente corta y ancha con carillas articulares bien definidas. El proceso espinoso de la séptima vértebra es alto, mientras que las demás no presentan. El foramen transverso se observa hasta la sexta cervical (Figura 2).

$1^{\circ}, 4^{\circ}$ y $13^{\circ}$ torácicas. Se tiene 13 vértebras torácicas. Presentan la característica de un hueso completo con un aumento del proceso espinoso proyectándose caudalmente hasta la toráxica 4 y declinándose hasta la torácica 12. Presentan una fóvea costal ligeramente cóncava. Apófisis mamilar poco prominente, la vértebra 12 es la anticlinal.

$1^{\circ}, 4^{\circ}$ y $6^{\circ}$ lumbares. Seis vértebras lumbares, presentando una estructura ósea completa con un ángulo abierto entre el cuerpo y el proceso transverso. El proceso espinoso se dirige hacia craneal con una forma ganchosa de las lumbares 4,5 y 6 .

Sacro. Estructura ósea bien definida. Presenta cinco vértebras fusionadas, con una forma triangular, un canal medular bien definido y las crestas laterales definidas y ligeramente afiladas.

Coccígeas. Son en número de siete. Las primeras vértebras mantienen ciertas características de una vértebra y las últimas casi cilíndricas.

\section{Costillas y Esternón}

Costilla. Huesos completos con carillas articulares bien definidas. Las últimas son delgadas.

Esternón. Hueso completo de estructura aplanada dorso ventralmente y con aumento de la densidad ósea que va desde el xifoides 


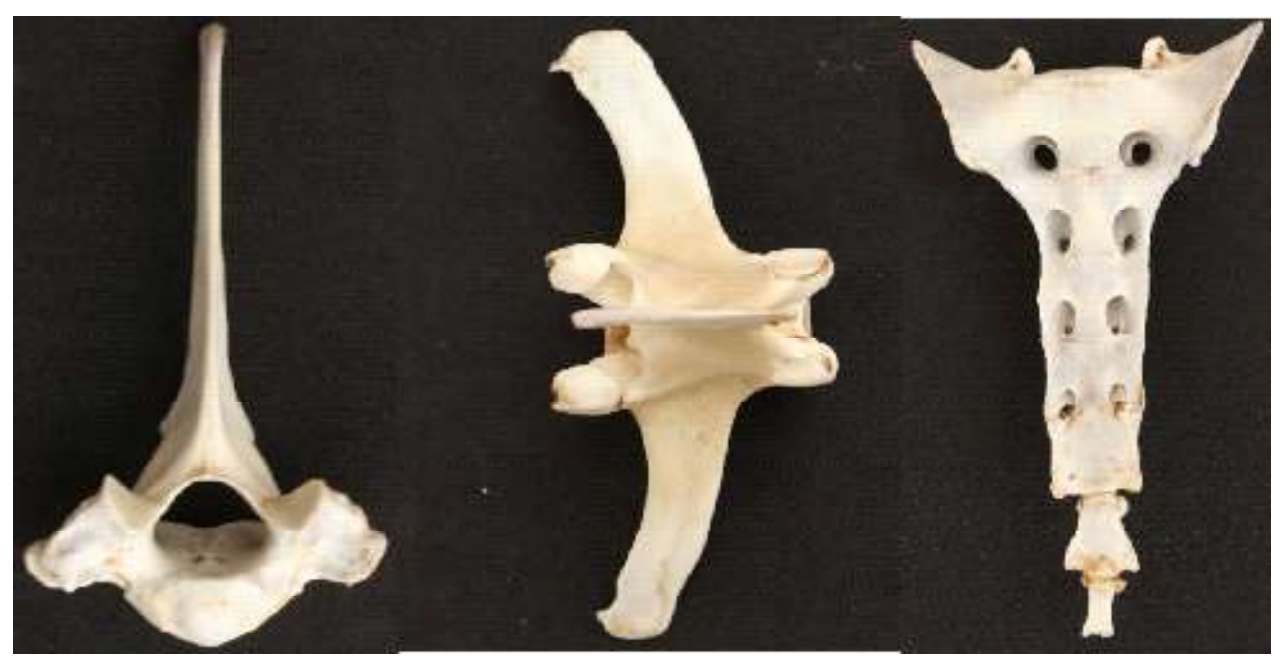

Figura 3. Detalle de las vértebras torácicas (T4, izquierda), lumbares (L3, centro) y del sacro (derecha, vista ventral)

al manubrio. Presenta siete esternebras con apófisis xifoides pronunciada (Figura 4). La cara ventral cóncava de un lado para el otro $\mathrm{y}$ en conjunto la superficie ventral convexa de craneal a caudal.

\section{Discusión}

La ausencia de huesos cigomáticos sobresalientes y los huesos maxilar e incisivos alargados, sugiere que la fuerza masticatoria es menor y el corte de los pastos sea débil, pero a la vez rápido, permitiéndole una mejor visión en el momento de alimentarse (Merino et al., 2005). A nivel del hueso lagrimal la presencia de una fosa profunda correspondería a la ubicación de la glándula preorbital, la cual es muy especializada (Barrio, 2010; Masters y Flach, 2015). En los cérvidos esta glándula tiene un papel olfativo e incluso visual (Ceacero et al., 2014). El caso de la fisura cubierta de cartílago también se observa en Capreolus capreolus al igual que en la cabra (Onuk et al., 2013).
Las piezas dentarias en la mandíbula y maxilar se encuentran bien implantadas, lo cual permite relacionarlo con la masticación. Es así que los ciervos macho presentan mayores requerimientos nutricionales, por ejemplo, para el crecimiento de las astas (Dryden, 2016). También la presencia de un diente canino a nivel del maxilar es una característica que no se presenta en todos los especímenes (Barrio, 2013), tal como se describe en Capreolus capreolus (Chaplin y Atkinson, 1968). La presencia de cuernos en el hueso frontal de los machos es importante, su crecimiento está relacionado a la testosterona y a la época de apareamiento, llegando a caerse posteriormente (Masters y Flach, 2015). A diferencia de los rumiantes domésticos, la apófisis que forma el asta en la taruca no presenta un cuello a nivel de la base ni presenta en su interior espacios irregulares como se describe en el ovino (Sisson et al., 1990a) ni tampoco se observa una cubierta cornea. La presencia de agujeros supraorbitarios puede servir para identificar al Hippocamelus antisensis, quién presenta por lo menos tres agujeros supraorbitarios 


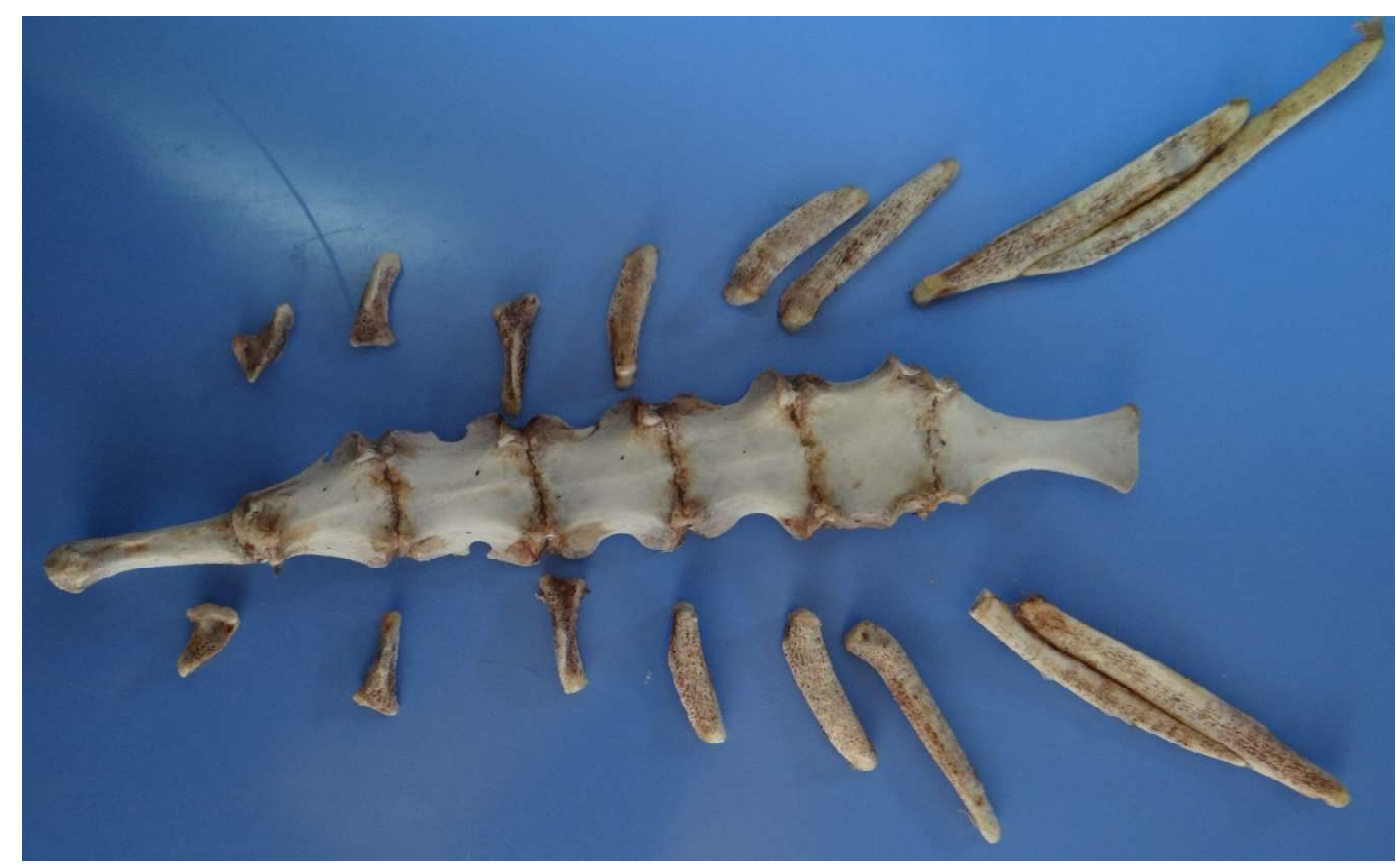

Figura 4. Detalle del esternón y los cartílagos costocondrales de la taruca (Hippocamelus antisensis)

(Pinto et al., 2016) en tanto que Hippocamelus bisulcus solo presenta un gran agujero supraorbitario (Groves y Grubb, 2011) y Capreolus capreolus que presenta de tres a cuatro agujeros (Onuk et al., 2013).

En algunos especímenes la apófisis nasal del hueso incisivo no se llega a relacionar con el hueso nasal, característica similar en los ovinos; sin embargo, en otras especies llega a unirse, como es el caso de Capreolus capreolus (Onuk et al., 2013). Asimismo, el hueso vómer se proyecta hacia caudal inclusive sobresaliendo del hueso palatino, característica que se corrobora en el Ozotoceros bezoarticus, que indica que las coanas están divididas por el septum nasal (Vazquez et al., 2018).

Con respecto a las vértebras, el género Cervus tiene la característica que las apófisis espinosas aumentan su altura hasta la torácica 4 para disminuir hasta la torácica 13 y mantenerse uniforme en la zona lumbar
(Kumar et al., 2000). Por otro lado, coincide el número de las vértebras torácicas, lumbares, sacras vértebras fusionadas) con lo descrito por Rajani et al. (2013) en el ciervo Axis axis. La mayoría de las características descritas de las vértebras son similares a lo descrito para rumiantes menores (Sisson et al., 1990b)

\section{Conclusiones}

- La cabeza es alargada por las características de los huesos maxilar e incisivo.

- La presencia de forámenes y las características de las astas en el hueso frontal pueden ser utilizados para su identificación

- La presencia de la fosa del lagrimal y la fisura cubierta de cartílago la diferencia de otras especies de cérvidos.

- Las coanas se encuentran divididas por el hueso vómer. 


\section{Agradecimientos}

Se agradece al personal de SERFORATFFS Cusco por las facilidades brindadas.

\section{Literatura Citada}

1. Agustina N, Ovejero Ovejero R, Cristóbal L, Ojeda R. 2020. Alpine mammals of South America. In: Encyclopedia of the world's biomes. Elsevier. p 441-460

2. Barrio J, Nuñez A, Pacheco L, Regidor H, Fuentes-Allende N. 2017. Hippocamelus antisensis. The IUCN Red List of Threatened Species doi: 10.2305/IUCN.UK.2017-2.RLTS.T10053A22158621.en

3. Barrio J. 2010. Taruka Hippocamelus antisensis (d'Orbigny 1834). In: Duarte JMB, Gonzalez S (eds). Neotropical cervidology: biology and medicine of neotropical deer. FUNEP. $\mathrm{p}$ 77-88.

4. Barrio J. 2013. Hippocamelus antisensis (Artiodactyla: Cervidae). Mammalian Species 45: 49-59. doi: 10.1644/901.1

5. Ceacero F, Bartošová $J$, Bartoš $L$, Komárková M, Ceacero F, LandeteCastillejos T, García AJ, et al. 2014. Habituating to handling: factors affecting preorbital gland opening in red deer calves. J Anim Sc 92: 4130-4136. doi: 10.2527/jas.2014-7716

6. Chaplin R, Atkinson J. 1968. The occurrence of upper canine teeth in roe deer (Capreolus capreoh) from England and Scotland. J Zool 155: 141144. doi: $10.1111 / \mathrm{j} .1469-7998.1968$.tb03034.x

7. Dryden GML. 2016. Nutrition of antler growth in deer. Anim Prod Sci 56: $962-$ 970. doi: 10.1071/AN15051

8. Fandos P, Orueta F. 1991. Variaciones morfológicas en el cráneo del corzo (Capreolus capreolus L). Doñana, Acta Vertebrata 18: 159-171.
9. Fuentes-Allende N, Vielma A, Paulsen K, Arredondo C, Corti P, Estades CF, González BA. 2016. Is human disturbance causing differential preference of agricultural landscapes by taruka and feral donkeys in high Andean deserts during the dry season? J Arid Environ 135: 115-119. doi: 10.1016/j.jaridenv.2016.08.018

10. Gazzolo C, Barrio J. 2016. Feeding ecology of taruca (Hippocamelus antisensis) populations during the rainy and dry seasons in central Peru. Int J Zool 2016: ID 5806472. doi: 10.1155/2016/ 5806472

11. Groves C, Grubb P. 2011. Ungulate taxonomy. Baltimore: Johns Hopkins University Press. 309 p.

12. Jackson J. 1973. Mandibular dental abnormalities in roe deer (Capreolus capreolus) from the New Forest. J Zool 177: 491-493. doi: 10.1111/j.14697998.1975.tb02250.x

13. Kumar N, Kukreti S, Ishaque M, Mulholland R. 2000. Anatomy of deer spine and its comparison to the human spine. Anat Rec 260: 189-203. doi: 10.1002/1097-0185(2000-1001)260:2<189::AID-AR80>3.0.CO;2-N

14. Masters NJ, Flach E. 2015. Tragulidae, Moschidae, and Cervidae. In: Fowler's zoo and wild animal medicine. Vol 8. Elsevier. p 611-625.

15. Merino ML, Milne N, Vizcaíno SF. 2005. A cranial morphometric study of deer (Mammalia, Cervidae) from Argentina using three-dimensional landmarks. Acta Theriologica 50: 91-108. doi: 10.1007/BF03192622

16. Merkt J. 1985. Social structure of Andean deer (Hippocamelus antisensis) in southern Perú. MSc Thesis. Vancouver, Canada: University of British Columbia. 143 p.

17. Onuk B, Kabak M, Atalar K. 2013. Anatomic and craniometric factors in differentiating roe deer (Capreolus capreolus) from sheep (Ovis aries) and 
goat (Capra hircus) skulls. Arch Biol Sci 65: 133-141. doi: 10.2298/ ABS1301141M

18. Pinto CM, Soto-Centeno JA, Núñez Quiroz AM, Ferreyra N, DelgadoEspinoza F, Stahl PW, Tirira DG. 2016. Archaeology, biogeography, and mammalogy do not provide evidence for tarukas (Cervidae: Hippocamelus antisensis) in Ecuador. J Mammal 97: 41-53. doi: 10.1093/jmammal/gyv151

19. Rajani CV, Sajitha IS, Pradeep M, Chungath JJ. 2013. Morphological studies on the thoracic, lumbar, sacral and coccygeal vertebrae in spotted deer (Axis axis). Tamilnadu J Vet Anim Sci 9: 113-121.

20. Ratcliffe PR. 1970. The occurrence of vestigial teeth in badger (Meles meles), roe deer (Capreolus capreolus) and fox (Vulpes vulpes) from the county of Argyll, Scotland. J Zool 162: 521-525. doi: 10.1111/j.1469-7998.1970.tb01288.x

21. Roe NA, Rees WE. 1976. Preliminary observations of the taruca (Hippocamelus antisensis: Cervidae) in southern Peru. J Mammal 57: 722-730. doi: $10.2307 / 1379442$

22. Schaller O. 1996. Nomenclatura anatómica veterinaria ilustrada. Acribia. $622 \mathrm{p}$.

23. [SERFOR] Servicio Nacional Forestal y de Fauna Silvestre. 2016. Primer informe parcial de inventario nacio- nal forestal y de fauna silvestre. [Internet]. Disponible en: https://sinia.minam.gob.pe/documentos/primer-informe-parcial-inventario-nacional-forestal-faunasilvestre

24. Sisson S, Getty R, Grossman J. 1990a. Anatomía de los animales domésticos. Tomo 2. $5^{\circ}$ ed. Elsevier Masson.

25. Sisson S, Getty R, Grossman J. $1990 b$. Anatomía de los animales domésticos. Tomo I. $5^{\circ}$ ed. Elsevier Masson. 1416 p.

26. Vazquez N, König HE, Jerbi H, Pérez $W .2018$. Gross anatomy of the nose, nasal cavity and larynx in the pampas deer (Ozotoceros bezoarticus, Linnaeus 1758). Anatomical Sci 1: 1-13. doi: 10.24294/as.v1i2.617

27. Villarroel MA, Troncoso N. 2017. Combinación de osteotecnia más conservación de músculos en montaje único de Canis lupus familiaris. Int J Morphol 35: 351-356. doi: 10.4067/S071795022017000100055

28. von den Driesch A. 1968. A guide to measurement of animal bones from archaeological sites. Peabody Museum Press. 148 p.

29. Weber M, Gonzalez S. 2003. Latin American deer diversity and conservation: a review of status and distribution. Ecoscience 10: 443-454. doi: 10.1080/ 11956860.2003.11682792 